\title{
EDITORIAL
}

\section{Petaflop supercomputers of China}

\author{
Guoliang CHEN (ه) \\ Department of Computer Science and Technology, University of Science and Technology of China, Hefei 230027, China \\ College of Computer and Software, Shenzhen University, Shenzhen 518060, China
}

(C) Higher Education Press and Springer-Verlag Berlin Heidelberg 2010

After ten years of development, high performance computing (HPC) in China has made remarkable progress. In November, 2010, the NUDT Tianhe-1A and the Dawning Nebulae respectively claimed the 1st and 3rd places in the Top500 Supercomputers List; this recognizes internationally the level that China has achieved in high performance computer manufacturing. The nation has already deployed and will deploy further supercomputers with computing capacities of hundreds of teraflops to petaflops in Beijing, Shanghai, Shenzhen, Tianjin, Jinan, and Changsha, which will greatly strengthen the supercomputing infrastructure. It should be noted that domestic high performance processors will be used in some of these systems. China has made significant advances in large-scale parallel computing areas such as low-power design, parallel programming, and reliability, as well as large-scale application software.

Dedicated to HPC efforts in China, this issue of Frontiers of Computer Science in China consists of papers from most major Chinese HPC vendors and research organizations. There are two survey papers, one of which by Xianghui Xie et al. elaborates on the evolution of supercomputers, while the other by Yunquan Zhang et al. provides a perspective of China's HPC development by examining China's HPC Top100 List. Four other papers focus on the technical aspects of three Chinese supercomputers. They cover: the heterogeneous architecture of Tianhe-1, by Xuejun Yang et al.; the design of the controller and communication system of the Dawning 6000, by Ninghui Sun et al.; and the balanced system design in DeepComp, by Mingfa Zhu et al. Finally, Zeyao Mo et al. present their research on JASMIN, a parallel programming infrastructure for scientific computing.

Recently, there have been at least three major factors driving the evolution of supercomputers: increasing concern for power and computation efficiency, and reliability, the rapid growth of data-intensive applications, and cloud computing. As a result, much work on HPC focuses on at least two major areas: heterogeneous architectures and general parallel programming models. In China, another driving factor of HPC is the use of domestic hardware and software. With the strong support of the Chinese government and the efforts of the entire HPC community, we confidently believe in a flourishing future for Chinese HPC.

E-mail: glchen@ustc.edu.cn

Guoliang CHEN is the guest editor of special section on Petaflop Supercomputers of China 